\title{
Strates
}

STRATES Matériaux pour la recherche en sciences sociales

13 | 2007

Paysage urbain: genèse, représentations, enjeux contemporains

\section{Fleuves en ville : enjeux écologiques et projets urbains}

\section{Sophie Bonin}

\section{(2) OpenEdition}

Journals

Édition électronique

URL : http://journals.openedition.org/strates/5963

DOI : $10.4000 /$ strates.5963

ISSN : $1777-5442$

Éditeur

Laboratoire Ladyss

Édition imprimée

Date de publication : 31 décembre 2007

ISSN : 0768-8067

Référence électronique

Sophie Bonin, « Fleuves en ville : enjeux écologiques et projets urbains », Strates [En ligne], 13 | 2007, mis en ligne le 22 octobre 2008, consulté le 08 septembre 2020. URL : http://journals.openedition.org/ strates/5963; DOI : https://doi.org/10.4000/strates.5963

Ce document a été généré automatiquement le 8 septembre 2020

Tous droits réservés 


\title{
Fleuves en ville : enjeux écologiques et projets urbains
}

\author{
Sophie Bonin
}

1 L'apposition de paysage et urbain se présente couramment comme une catégorie accompagnatrice, donc séparée, de celle de paysage naturel. Une revue de l'occurrence de cette expression paysage urbain dans l'Encyclopedia Universalis ${ }^{1}$ permet de le confirmer. On la rencontre à l'article « Patrimoine monumental »: «[...] les ayant un peu vite jugé désuets et inadaptés, on avait éliminé ou défiguré les éléments les plus précieux du paysage urbain ou naturel ». Cette distinction se retrouve dans les travaux classiques sur le paysage urbain, et jusqu'aux politiques en ce domaine, qui se relient peu à la question de la nature mais bien plus à celle du patrimoine, ou à celle de l'esthétique. La question du paysage est alors travaillée en ville de deux façons.

2 D'un côté, en termes de lecture, il s'agit, par analogie avec les paysages ruraux qui ont fait l'objet de travaux mieux individualisés, de mettre en évidence sa structure, ses lignes, ses volumes, ses matériaux, ses couleurs ${ }^{2}$. Mais contrairement aux paysages ruraux, il n'y a pas de considération pour les relations fonctionnelles avec l'environnement. Par exemple, en milieu rural, l'étude des Paysages de terrasses, de Régis Ambroise, Pierre Frapa, Sébastien Giorgis ${ }^{3}$, est d'abord préoccupée, et puise ses motivations, dans la mise en évidence de rapports "structures-fonctions » entre activités humaines et pente, par un système original de gestion de l'eau et des sols. Alors qu'en ville, les motifs jugés structurants ne sont pas ceux du milieu naturel, des données édaphiques notamment, mais la voirie, les infrastructures, c'est-à-dire des artefacts, et surtout des artefacts minéraux. Les usages sociaux qu'on peut y lire sont de plus très limités à certains domaines : les transports, les flux, notamment. Un article récent faisant la revue du paysage urbain dans les politiques d'urbanisme et d'environnement à partir des textes juridiques, confirme cette séparation de la question du paysage urbain d'avec les «fonctionnements sociaux et écologiques » et conclut sur la perspective possible de cette intégration, "y compris dans ses composantes biophysiques ${ }^{4}$ ». Il rappelle pour l'instant l'importance des visées patrimoniales et de la logique de protection. 
3 D'un autre coté, en termes de traitement de la nature, les projets de paysage en ville retiennent d'abord et quasi exclusivement une nature symbolique; et le végétal est conçu dans ces projets comme élément de décor, parmi d'autres, qui va s'ajouter postérieurement à la «table rase » incontournable qu'opèrent les projets. Un platane est planté comme on poserait une sculpture ; un mur est peint en vert, ou une façade vient symboliser une forêt : ces actions permettent « de réinventer » du paysage, mais il s'agit d'un travail esthétique qui joue des sensations et non d'une matérialité vivante, qui revendique d'ailleurs l'absence de fonctionnalités autres que symbolique ${ }^{5}$. Un architecte plasticien reconnu dans ce domaine, Bernard Lassus, a développé de façon théorique l'importance du « respect de l'existant », du substrat-support-apport ${ }^{6}$. Mais le quartier, l'îlot, l'immeuble, n'est pas conçu comme un biotope, ou appartenant à un biotope: les problématiques du sol, de la faune, la flore, l'air, l'eau, en termes de dynamique et de qualité, ne sont pas intégrées. Face à la montée de l'écologie, et à propos des fleuves, on a pu récemment voir dénoncer ce genre d'actions, poursuivies dans les années 1980 et 1990, comme étant une " gadgétisation du paysage fluvial ${ }^{7}$ ".

Ce n'est que bien plus récemment, mais cela prolonge ce dernier processus, que se sont développés des travaux sur la naturalité en ville, qui reprennent dans des directions nouvelles l'écologie urbaine, mais surtout à travers une idée de nature produite par la ville ${ }^{8}$. Il n'est peu ou pas fait mention d'objets naturels comme les fleuves, qui « vont de soi » au point qu'on ne les voit pas; qui relèvent également d'une échelle différente de celle du paysage, ou qui posent le paysage seulement comme une des nombreuses dimensions de la question de la nature, dimension qui intéresse peu les auteurs car le paysage y apparaît comme une « réduction du substrat naturel à ses intégrations dans la sphère anthropique ${ }^{9}$ ». Dans ces travaux, de façon classique, le fleuve apparaît comme une " brèche ouverte dans le tissu urbain ", posant le problème de la séparation entre les deux rives, et comme "phagocyté par les objets de sa domestication ${ }^{10}$ ». La récente réappropriation des berges, déjà bien amorcée et d'ailleurs constatée, n'entre pas dans la problématique étudiée. Le fleuve est seulement un des exemples illustrant une nature domestiquée, effacée physiquement et idéellement. On retrouve cette absence du fleuve comme nature dans un numéro spécial des Annales de la Recherche Urbaine consacré aux Natures en ville ${ }^{11}$, en pleine période de "reconquête » des fleuves urbains, mais où il n'en est fait aucune mention: c'est quasi exclusivement la végétation qui est naturelle en ville, ou alors il s'agit de nature dans un sens plus philosophique ou sociologique. La nature de l'urbaniste, ou même des sciences humaines n'est pas celle de l'écologue, ce qui constitue une limite, importante, à ce que nous développerons dans ce texte. Parmi les multiples sens possibles du fleuve comme nature, nous en avons exploré deux : le fleuve comme système écologique, ressources matérielles, fonctionnelles; et le fleuve (la nature) comme ressources immatérielles, esthétiques, sur une échelle qui va du simple décor à la relation vécue.

Il semble pourtant que les nombreuses opérations de reconquête, ou de retrouvailles, des villes avec le fleuve puissent nous interroger plus profondément, et fassent évoluer ces conceptions : cette nouvelle place n'indique-t-elle pas une évolution marquante des réflexions et des politiques en matière de paysage urbain? Cette valorisation ne montre-t-elle pas que la nature désormais participe largement à la sensibilisation sociale et politique sur le paysage urbain? N'est-ce pas l'entrée de l'écologique dans l'esthétique, l'ouverture d'une voie pour des usages socioéconomiques porteurs d'un projet de paysage, donnant tort aux discours se désolant de notre incapacité à joindre 
ces deux «bouts » du pays-paysage ? Cette évolution enfin interroge la confusion entre valeurs écologiques et valeurs paysagères, qu'Alain Roger dénonçait comme une « verdôlatrie ${ }^{12}$ » dangereuse, empêchant de régler les problèmes des unes comme des autres. Qu'en est-il ?

Nous reviendrons dans un premier temps sur l'idée d'émergence d'un " paysage naturel urbain », en examinant en particulier dans quelle mesure l'écologie est invoquée dans les opérations de réappropriation des fleuves par les villes. Notre acception de l'écologie inclut les écosystèmes au sens strict (les relations biotope-biocénose) mais aussi les activités humaines (nouveaux usages, nouvelles dynamiques socioéconomiques), le principal étant une mise en relation et en équilibre interactif, de ces éléments. Dans un second temps, nous questionnerons ce paysage naturel urbain comme enjeu de nouveaux modèles de développement (nouveaux usages, nouvelles dynamiques socioéconomiques). Comment le fleuve est-il travaillé dans ces projets de «reconquête»? Est-ce une valorisation esthétique, symbolique, ou écologique? ou l'émergence de nouvelles pratiques sociales en milieu urbain? Nous nous appuierons sur l'étude de la place du fleuve dans deux projets urbains (Rhône en aval de Lyon; Loire à Nantes).

Paysage naturel... urbain ? La ville, participant aux logiques de protection, de gestion, et de mise en valeur d'écosystèmes?

7 Il faut noter que les opérations les plus exemplaires de reconquête des cours d'eau, au nom du paysage notamment (mais pas seulement), sont le fait des villes, dans un mouvement qui apparaît comme généralisé, se déroulant au même moment dans beaucoup des grandes villes riveraines, d'Europe et d'ailleurs. Il n'est plus réaliste aujourd'hui de prétendre dresser la liste des villes riveraines qui ont élaboré, des années 1990 à aujourd'hui, des Plan Bleu, Livre blanc pour l'aménagement des berges, Opération plage, restauration des quais, etc. Des travaux abondants en témoignent en France, dès la fin des années 1980, qui concernent la Seine, la Loire et le Rhône surtout ${ }^{13}$ ; mais on peut aussi citer le cas de Londres ${ }^{14}$, ou de Philadelphie ${ }^{15}$. Les espaces fluviaux sont arrivés dans les réflexions des urbanistes depuis vingt ans environ (commission «Lyon, ville fluviale » constituée en 1981 ; colloque La ville et le fleuve à Lyon, 1987; Mission fleuve réalisée par Michel Cantal-Dupart à la demande du ministère des Transports). Encore récemment, on peut citer un numéro de Diagonale «De berges en rives " (nov.-déc. 2003), un colloque en septembre 2003 à Orléans Le fleuve et ses territoires. Des enjeux patrimoniaux aux grands projets urbains, ou l'activité débordante de l'association La Seine en partage, créée en 2001. Cette mobilisation nous interroge : d'où vient-elle ? On y voit facilement, en première hypothèse, la montée de la sensibilité écologique, mais qu'en est-il en termes d'actions et de perspectives? Nous avons particulièrement travaillé deux cas, qui se trouvent être souvent cités comme pionniers dans ce type d'aménagement : Lyon et Nantes.

Une grande diversité de contextes pour des projets semblables

On est face à une demande des villes qui apparaît nouvelle, encore très mal cadrée, et les témoignages reflètent la difficulté de ces situations multiformes, dans ses enjeux, dans les lieux eux-mêmes, dans les échelles d'intervention.

$\mathrm{Du}$ point de vue des lieux d'abord, des relations propres à chaque ville existent ou se sont nouées entre citadins et fleuve, liées à l'histoire et à la conformation des rives, aux pratiques sociales et économiques, à l'accessibilité. Les berges sont des terre-pleins naturels ou des quais maçonnés, bas ou hauts; les risques d'inondation sont plus ou 
moins forts. Elles ont un statut foncier variable, mais qui peut être assez contraignant (privés ; Domaine public fluvial ; contrats divers d'occupation, pour l'agriculture ou la chasse). Enfin, ces sites riverains ont une place dans l'imaginaire qui peut être très variée, négative ou positive, guinguettes ou bas-fonds, opposant plus ou moins fortement, mais assez systématiquement rive gauche et rive droite.

$10 \mathrm{Du}$ point de vue des échelles d'intervention, les projets se portent sur un réaménagement de quai dans un village, jusqu'à un projet intercommunal, départemental voire transrégional et européen. Mais la place des fleuves a d'abord émergé, souvent, dans les textes généraux d'orientation de l'urbanisme, les Schémas directeurs d'aménagement et d'urbanisme (SDAU). Les institutions qui ont le plus l'initiative de ces projets peuvent aussi bien être très locales (les communes du Sud de Lyon par exemple), que les gros établissements des agglomérations (la Courly, Communauté urbaine de Lyon), et des institutions de gestion de l'eau et de l'aménagement $\mathrm{du}$ fleuve (Compagnie nationale $\mathrm{du}$ Rhône). Toutes ont pu être influencées et poussées par les institutions de l'État chargées de la protection et mise en valeur de l'environnement, en propre (directions régionales de l'environnementDiren) ou parce qu'elles sont chargées de la gestion de l'eau (Agences de l'eau).

11 Enfin, du point de vue des enjeux, les objectifs des municipalités sont très variés, mais relativement convergents. Le plus souvent, il s'agit de transformer les berges en un espace public convivial ou touristique (promenade piétonne, ou en gabares : Albi). Mais c'est aussi élaborer une "trame verte " à partir des berges (Orléans), et valoriser l'image de la ville; avec notamment la création de parcs. C'est aussi l'occasion parfois d'agrandir le centre-ville en réinvestissant des friches industrielles et portuaires, intégrant des équipements publics, culturels ou de loisir (Greenwich, Nantes). Le contexte de ces espaces dans les années 1980 était souvent une reconversion économique vers des activités tertiaires, culturelles ou ludiques. On peut citer par exemple la reconquête des quais à Bordeaux qui est un volet du projet urbain global, d'ouverture sur la Garonne mais aussi de découverte de la rive droite; ou bien celle des bords de Loire à Nantes, avec les 10000 habitants de l'île Beaulieu/île Sainte Anne/ prairie au Duc, exemple qui sera développé plus loin. L'enjeu est parfois plus important et devient source de conflits: il s'agit alors de transformer une vocation actuelle, "active" des berges, en une vocation plus douce, qui prend les atours du " développement durable ", d'un modèle d'aménagement " doux ». Par exemple à Lyon, ce sont cinq $\mathrm{km}$ de quais, auparavant utilisés pour le stationnement, qui ont été réaménagés pour les piétons et cyclistes. À Paris, le contexte est plus délicat encore car cette fois la circulation automobile est en jeu, d'où la méthode d'occupations temporaires et festives. On est là au cœur du jeu actuel dans des changements de représentation, voire même des changements de modèle de développement. D'un côté, on retrouve les problématiques $d u x^{e}$ siècle, sur les échanges, la vie économique, le travail, les emplois... la voiture, c'est-à-dire des problèmes de gestion de flux surtout ; de l'autre se sont développées les problématiques de l'environnement, des loisirs, du cadre de vie, c'est-à-dire des problèmes de gestion des espaces. Il n'est pas acquis que le développement durable soit d'un côté plus que de l'autre, même si c'est le deuxième modèle qui se drape le plus dans ces discours. On ne peut donner complètement tort à des commentaires sur ces nouveaux aménagements des berges comme celui de Claude Prelorenzo :

[...] ces projets sont timides, voire muets en ce qui concerne les usages de l'eau. On peut se demander s'il est possible de reconquérir des berges désertées, de 
réinventer une nouvelle ville en relation avec l'eau en laissant la rivière, le lac ou le bord de mer dans leur état actuel de stérilité, en leur affectant un médiocre statut d'espace libre ou plus vaguement encore d'espace naturel ${ }^{16}$. dire le conflit entre écologie et esthétique. Ils disent aussi le problème de fond de toutes les actions en matière de paysages, et là il en est de même pour les paysages ruraux : quelle durabilité économique pour ces investissements symboliques ou plus globalement idéels ? Quel sens à ces pratiques dépourvues de productivité tangible?

Le fonctionnement des écosystèmes introduit dans les préoccupations urbanistiques? Le cas des fleuves, berges, îles...

13 l'étude du paysage d'un sens de décor, signification formelle et esthétique, vers une signification en termes de cadre de vie, et pour cela l'émergence des soucis écologiques et sociaux est certainement moteur; un moteur parfois contradictoire ou du moins ambiguë, mais quand même... Il convient en effet de revenir sur le contexte de ces actions, voir les «paysages » concernés initialement, pour mieux comprendre la place attribuable à l'écologie. On constate en effet que, contrairement à la diversité des situations possibles de paysages fluviaux urbains que l'on vient d'évoquer, les sites, qui ont fait le plus l'objet de réaménagement et de projet de grande ampleur, ont des caractéristiques communes : ce sont, ou c'étaient, des paysages en crise ; et ce sont des espaces de disponibilité foncière, ou des espaces saisis par la gestion des risques.

Que ce soit à Nantes, à Lyon, en amont de Paris, l'attention portée au fleuve vient du problème posé à la planification urbaine institutionnelle de la gestion des friches industrielles. Les secteurs fluviaux concernés par cet urbanisme de prise en compte de la nature correspondent assez fidèlement aux espaces que l'on désignait dans les années 1980 comme des "paysages en crise ", voire comme des espaces annonciateurs de la «mort du paysage». Le titre d'un colloque et d'un ouvrage, avec un point d'interrogation final ${ }^{17}$, évoquait précisément la transformation des territoires du fait d'une urbanisation mal maîtrisée, de l'industrialisation, des zonages. Les auteurs s'interrogeaient alors sur le fait qu'il y avait une perte d'humanité, une perte de sociabilité, dans certains territoires qui ressemblent fort à ceux que l'on a pu explorer en Seine Amont par exemple ${ }^{18}$. était très justifié, et que la question de la reconnaissance d'un paysage ne se décrétait pas à une époque donnée. Le temps seul pourrait dire si ces paysages industriels, ces paysages de la banlieue ou des parkings pourraient être acceptés et appréciés, un jour, par certains.

Aujourd'hui, de ce point de vue, trois choses ont changé : patrimonialisation ", et la mise en place de politiques culturelles autour de l'industrie. Certaines installations, certains bâtiments, sont conservés à titre symbolique ou reconvertis à titre ludique: l'IBA d'Emscher Park est à ce titre un modèle qui a largement été médiatisé ${ }^{19}$.

18 Les friches ont dégagé du foncier et ouvert un espace de liberté. Elles fournissent l'occasion de repenser les fonctions économiques et sociales, voire l'identité de ce territoire (pour une réflexion prospective). Il en est de même du Domaine public fluvial (DPF), qui n'est plus entretenu par l'État depuis le déclin de la navigation : depuis la loi 
du 30 juillet 2003, relative à la prévention des risques naturels et technologiques, l'État est autorisé à transférer gratuitement une partie de son domaine public fluvial aux collectivités territoriales qui le souhaitent. Les enjeux stratégiques qui autrefois maintenaient l'État en position très défensive quant à l'occupation du DPF ne sont plus. La gestion des risques et le renforcement de la réglementation dans ce domaine a aussi renforcé la nécessité d'une réflexion sur le devenir de ces espaces. Le discours scientifique sur les bienfaits d'une gestion douce des inondations, qui passe par la recréation de champs d'expansion des crues plutôt que par la création de nouveaux barrages, a joué son rôle. La mise en place des plans de prévention des risques d'inondation (PPRI) a permis une prise de conscience, suivie d'une prise en compte concrète de l'hydraulique naturelle du fleuve.

Dans ce faisceau d'éléments moteurs du changement, on voit donc que le contexte n'est pas tant celui d'une reconnaissance de valeurs écologiques, mais de changements économiques fondamentaux, qui amènent la recherche d'un nouveau modèle de développement, d'une autre forme de valorisation (économique) de ces espaces fluviaux. Mais ces différents changements convergent pour une reconnaissance de ces espaces comme combinant valeurs paysagères et potentiel écologique.

21 Nous allons donc dans une deuxième partie examiner cette nouvelle dynamique socioéconomique, attendue par ces projets.

Nouveaux usages, nouvelles dynamiques socioéconomiques : le paysage naturel urbain comme enjeu de modèles nouveaux de développement ?Des commissions, des chartes, des plans... et leur concrétisation

Deux exemples (le Rhône en aval de Lyon; la Loire à Nantes) permettent de voir se construire et évoluer les projets de reconquête, dans leurs objectifs concrets, et dans leur gouvernance: ainsi peut-on observer les décalages entre discours, réalisations projetées, et réalisations accomplies. Ces deux tableaux (voir ci-dessous) ont également pour objectif de mettre en parallèle la chronologie des changements sur ces deux territoires. (Cf. tableaux en documents annexes.)

23 En gras ont été précisés les objectifs réalisés, lorsqu'il s'agissait de réalisations concrètes et non de déclarations d'intention. En encadré plus épais, les lignes correspondent à des événements importants à l'échelle du bassin versant ou de l'agglomération lyonnaise, qui ont influencé les changements de la place du fleuve pour les collectivités locales ${ }^{20}$. Il était nécessaire, dans ce second registre, de faire une difficile sélection dans un historique touffu et complexe, sur le Rhône comme sur la Loire. On gardera à l'esprit qu'un tel exercice est donc très réducteur : ce sont plutôt quelques repères pour situer l'histoire de la « reconquête » sur nos deux sites.

Cette double chronologie montre d'abord nettement que l'on change, très rapidement, en moins d'un demi-siècle, de modèle de développement à l'échelle des principes généraux d'aménagement. On est passé d'une logique de développement sectoriel et d'intégration des différents secteurs économiques institutionnalisés (navigation, énergie, irrigation, surtout) à la mise en avant d'une logique de requalification d'espaces naturels, de réouverture au public, finalement de revalorisation paysagère. La première logique est mise en œuvre de façon rapide et efficace sur le Rhône, mais va beaucoup s'attarder sur la Loire; permettant d'autant plus à la seconde logique de s'installer dans le bassin ligérien, avec des succès médiatiques connus. Mais ces tableaux montrent aussi d'autres logiques à l'œuvre à l'échelle locale, celle des aménagements concrets. Les deux contextes initiaux sont là assez différents : dans le 
cas des îles rhodaniennes, il s'agit de mettre en valeur des espaces agricoles en friche, alors qu'à Nantes, l'île est un espace largement industrialisé au XIX siècle. Les premières sont plus nettement des espaces ruraux, quoique les deux situations soient proches de métropoles très dynamiques. Mais au final, les options exprimées pour ces espaces se rejoignent et s'avèrent donc souvent en décalage avec les principes plus globaux, ce que l'on analysera mieux dans la partie suivante : la volonté d'équipements, de loisirs ou culturels, domine largement les volontés de préservation ou de restauration écologique.

Le décalage entre discours et projets

Au final, la comparaison de ces deux situations nous montre que la reconquête du fleuve, à travers le réinvestissement de ces îles, vient d'abord d'accords permis par le désinvestissement industrie : il fédère des acteurs jusqu'à présent en conflit d'intérêt ; il concilie une stratégie d'agglomération (entrée sud du Lyon international, densification du centre ville pour Nantes, image attractive) et le développement communal. Un discours global, qui vient d'en haut comme on le voit (Plan Rhône, Plan Bleu; Plan Loire Grandeur Nature sur la Loire), met en avant un nouveau modèle de représentations du fleuve, soucieux des milieux naturels et d'un accord entre usages et dynamiques écologiques, au tournant des années 1990. Il est facilement intégré localement: le fleuve y apparaît comme bien collectif, à valoriser sur un plan symbolique, perceptif, récréatif. Mais la «logique de rente ${ }^{21}$ " subsiste dans toutes les initiatives concrètes. Encore cela pourrait ne pas être incompatible avec une prise en compte du fleuve comme biotope. Mais en dehors d'actions de protection bien délimitées spatialement, ou de revégétalisation, les actions mises en place en ville ne témoignent pas d'une intégration de l'écologie fluviale. Les projets s'articulent d'abord et toujours autour de la construction d'équipements, et d'une mise en espace vert de ces espaces naturels. Sur le Rhône, plus en amont, dans Lyon cette fois, sous le titre "Lyon redonne vie à sa presqu'île ${ }^{22}$ ", on expose comment la cité va remédier à des décennies où «elle a tourné le dos au Rhône et à la Saône, sous exploitant ce patrimoine naturel exceptionnel »: ce sera la construction d'un ensemble immobilier (des immeubles «juste au bord de la darse»), un centre commercial avec multiplexe cinématographique qui sera la pierre angulaire du projet, un nouveau siège pour le conseil régional $\left(38000 \mathrm{~m}^{2}\right)$, etc. Sur l'île de Nantes, l'enjeu est de façon similaire la construction de plus de $800000 \mathrm{~m}^{2}$ de logements, bureaux et équipements publics d'ici 2007, de faire de l'île Beaulieu un centre commercial urbain et non périurbain, c'est-à-dire de densifier la ville, luttant contre l'étalement dont souffre particulièrement l'agglomération nantaise ${ }^{23}$. Il s'agit de "faire de l'île de Nantes le cœur de l'agglomération nantaise du xxI siècle " ( Nantes demain », 2005). La démarche a effectivement changé : respect de l'existant, prise en compte au moins visuelle de la présence du fleuve, et mise en valeur du patrimoine ${ }^{24}$. Des aspects économiques directs aussi sont apparus, en rapport avec la dimension visuelle en particulier (résidences et lofts « avec vue »). Mais la dimension écologique de la relation fleuve-ville n'est pas dans les préoccupations: l'eau (sa présence, sa circulation), l'énergie, les matériaux en relation avec le fleuve ne sont pas intégrés. Seule la végétation participe parfois au projet, mais dans le seul cadre du traitement des espaces verts. Ainsi sur l'île de Nantes, pour le jardin de l'île Mabon, on laisse la végétation poursuivre son développement sur une friche industrielle, sans plantation. La mission d'urbanisme confiée au paysagiste "porte sur la mise en valeur du patrimoine ${ }^{25}$ ", et non sur la possible relation à la nature permise par la proximité du 
fleuve. La Loire est quand même bien utile au projet, formellement, car elle lui permet d'afficher « 400 ha d'espaces publics » (l'île en faisant 350), car 245 ha de fleuve autour de l'île sont inclus dans cette surface ${ }^{26}$. C'est ainsi que le fleuve est l'objet d'un tour de passe-passe aux dépens des espaces publics, et sans qu'apparaisse pour autant une réflexion sur l'utilisation possible de la Loire par les habitants actuels et futurs. "redonnent vie " à leur fleuve, "se réconcilient avec ", ou «partent à le reconquête de». Il nous conduit notamment à infirmer l'hypothèse d'un lien entre cette dynamique et une montée des préoccupations environnementales; l'hypothèse qu'il s'agit d'une pénétration de la nature, des préoccupations pour les hydroécosystèmes comme milieu de vie, en ville. Berges «naturelles ", îles, ripisylves, eau de qualité, de même que les usages que l'on voit soutenir les projets en matière d'ingénierie écologique (pédagogie de l'environnement, loisirs de proximité comme la pêche ou la baignade) ne sont pas les objets de cette reconquête. Ce type de préoccupations, que l'on voit effectivement motiver des projets dans les espaces ruraux, et qui sont parfois dans les discours programmatiques, disparait très tôt dans la mise en forme des deux projets urbains étudiés. Il semble souvent aussi venir de haut, d'un discours nouveau des grands corps d'ingénieurs notamment, qui mériterait d'être mieux exploré, et des idées soutenues par le ministère de l'Environnement (écologie) et ses DIREN. Il semble bien entendu localement, mais sa mise en œuvre se heurte à d'énormes obstacles. Les logiques réglementaires des actions en matière de paysage urbain, restent préoccupées d'abord de protection, de conservation du patrimoine, voire de " patrimonialisation ${ }^{29}$ ". Cette valorisation du fleuve en ville vient bien en apparence de la montée des préoccupations environnementales, de la réintroduction d'une place de la nature en ville; mais elle ne prend place qu'au titre d'un visuel, et d'une symbolique vectrice d'identité. Le fleuve sert à changer une identité dévalorisée d'espaces de friches agricoles ou industrielles notamment, mais sans rien changer aux pratiques traditionnelles d'urbanisation.

Les enjeux esthétiques que véhicule la notion de paysage urbain ne sont pas transformés par cette émergence du fleuve comme nature; le fleuve ne parvient pas 
véritablement à catalyser une relation nouvelle entre une demande sociale, des usages économiques, et la valorisation d'un milieu particulier.

Mais cette conclusion ne doit pas sembler négative en termes de perspectives de recherche. Tout d'abord, seulement deux situations particulières ont été réellement détaillées. Ensuite, les sources utilisées restent très institutionnelles, documents de planification et études de projet essentiellement : conclure réellement sur notre sujet aurait nécessité une approche anthropologique différente, à partir d'enquêtes sur les représentations et les pratiques des acteurs de l'aménagement. Le fleuve est resté dans notre approche un espace abstrait, éventuellement construit comme territoire, mais un espace inexploré en tant que nature, que globalité écologique. Le fleuve est apparu à travers nos sources essentiellement, au mieux, comme un décor; mais c'est aussi que les plans et études d'urbanisme sont aux prises avec des contraintes et des tensions considérables. Il conviendrait donc d'explorer cette question avec d'autres méthodologies.

Enfin, beaucoup de discours et de signes annoncent des changements en cours. Un des signes les plus clairs, sans que l'on puisse dire si cela bouleversera le statut des fleuves, est la prise en main régionale et locale des questions qui les touchent, et la recherche d'une nouvelle gouvernance. Les enjeux actuels, définis comme on l'a vu largement par l'État («bon état écologique»; «ressource environnementale» dans sa dimension aussi de bon fonctionnement hydraulique en prévision des crues; économie résidentielle, loisirs, services culturels, sportifs, etc.) ne sont pas considérés comme nationaux, du ressort du pouvoir central. Les préoccupations environnementales sont donc renvoyées à l'efficience sur ces sujets de la décentralisation (qui peut aussi trouver de l'aide, sur ces thématiques, auprès des politiques européennes). Le chemin qui conduirait à un fonctionnement écologique de la ville au bord de son fleuve, à une harmonie entre images et usages du fleuve, s'il existe, est encore long.

\section{NOTES}

1. Édition numérique de 2002.

2. Voir les travaux de S. Rimbert, pionniers en France, mais inspirés des travaux anglosaxons : Les paysages urbains, Paris, Armand Colin, 1973 ( $1^{\text {re }}$ édition). Voir aussi plus récemment : J.-M. Loiseau, F. Terrasson, Y. Trochel, Le paysage urbain, Paris, Éditions Sang de la terre, 1993.

3. R. Ambroise, P. Frapa, S. Giorgis, Paysages de terrasses, Aix-en-Provence, Édisud, 1989.

4. N. Blanc, S. Glatron, « Du paysage urbain dans les politiques nationales d'urbanisme et d'environnement ", L'Espace géographique, $\mathrm{n}^{\circ}$ 1, 2005, p. 65-80 (80).

5. B. Lassus, Couleur, lumière, paysage..., Paris, Monum, Éditions du patrimoine, Centre des monuments nationaux, 2004. Voir les pages 48 à 54, à propos de ses projets des années 1970 et 1980, son travail sur les couleurs, les contrastes.

6. Ibid., p. 163. 
7. Le fleuve fait surtout l'objet de décors à confectionner, dans les préconisations du premier Plan Bleu à Lyon en 1991 ; voir C. Gérardot, « Les élus lyonnais et leurs fleuves : une reconquête en question ", Géocarrefour, vol. 79, n 1, 2004, p. 75-83 (78).

8. N. Mathieu, M. Jollivet, Du rural à l'environnement : la question de la nature aujourd'hui, Paris, Arf, L'Harmattan, 1989 ; N. Blanc, N. Mathieu, « Repenser l'effacement de la nature dans la ville », Courrier du CNRS, La ville, mai 1996, p. 105-107 ; W. Hucy, La nature dans la ville et les modes d'habiter l'espace urbain - Expérimentation sur l'agglomération rouennaise, thèse de géographie, université de Rouen, 2002.

9. W. Hucy, op. cit., p. 38.

10. Ibid., p. 58.

11. Annales de la recherche urbaine, $\mathrm{n}^{\circ} 74,1997$.

12. A. Roger, Court traité du paysage, Paris, Gallimard, NRF, 1997, p. 134.

13. V. Claude, A. Guillerme, «La vallée de l'Orge-1880-1977 », Annales de la recherche urbaine, numéro spécial «L'eau dans la ville », 1986, p. 78-88; A. Guillerme, « Le testament de la Seine ", Revue de géographie de Lyon, n 65/4, 1990, p. 240-250 ; J. Labasse, "Réflexions d'un géographe sur le couple ville-fleuve », La ville et le fleuve, colloque du $112^{\mathrm{e}}$ Congrès national des sociétés savantes, Lyon, 21-25 avril 1987, 1989, p. 9-22 ;

J. Pelletier, «Sur les relations de la ville et des cours d'eau », Revue de géographie de Lyon, $n^{\circ}$ 65/4, 1990, p. 233-239 ; J. Bethemont, A. Vincent, « La revitalisation des fronts d'eau urbains dans la vallée du Rhône ", Revue de géographie de Lyon, n 73/4, 1998, p. 331-335.

14. C. Dalnoky, M. Desvigne, «Pixel d'arbres pour Greenwich », Pages, Paysages, $n^{\circ} 7$, 1998, p. 172-176; T. Erskine, "Housing, Greenwich, London-Eco urbanity », The Architectural Review, vol. 211, n 1259, 2002, p. 41-45.

15. B. Lassus, op. cit., p. 170-181.

16. C. Prelorenzo, « La ville au bord de l'eau ou les contemplations du vide », La ville, $n^{\circ}$ 2, Sur Seine, Le Havre, Rouen, Paris-banlieues, 1995.

17. F. Dagognet (dir.), Mort du paysage? Philosophie et esthétique du paysage, Seyssel, Champ Vallon, 1982.

18. F. Pousin (dir.), " Pré-étude Paysage industriel de la Seine Amont », Rapport de synthèse, Mission Seine Amont, Direction régionale de l'Équipement de l'Île-de-France, 2004.

19. L'« exposition internationale d'architecture » (l'IBA) d'Emscher Park (1989-1999) est un processus de réhabilitation d'une région socialement et écologiquement traumatisée par son passé industriel révolu. Le réseau hydrographique a servi de point d'ancrage à ce programme. Voir W. Siebel, «L'IBA ou l'exposition internationale d'urbanisme d'Emscher Park : une stratégie pour le renouveau économique, écologique et social d'une ancienne région industrielle ", Espaces et sociétés, n 72, 1993, p. 65-76; S. Schaffer, « La seconde jeunesse de la Ruhr », Urbanisme, n² 267-268, 1993, p. 90-93 ; le numéro spécial consacré à ces réalisations de la revue Topos - spécial IBA, European landscape magazine, n² 26, mars 1999 ; L'IBA Emscher Park : un anti-modèle, coll. « Projet urbain ", n² 21, Éditions DGUHC (ministère de l'Équipement), sept. 2000.

20. Ce tableau a été composé à partir des études disponibles, de revues de presse, des sites Internet. Pour Lyon et le Rhône, voir L. Cottet-Dumoulin, « Processus de requalification d'un espace «naturel » en marge d'une agglomération - Le cas des îles du Rhône aval au sud de Lyon ", in A. Vincent (dir.), Le monde alpin et rhodanien - Le Rhône. Un fleuve et des hommes, Éditions Centre alpin et rhodanien d'ethnologie, 1999, p. 175-192. Pour Nantes, voir A. Chasseriau, J.-P. Peyon, « Le projet île de Nantes ou 
comment la ville se réconcilie avec son fleuve ", Actes du FIG de Saint-Dié-des-Vosges, http://xxi.ac-reims.fr/fig-st-die/actes 2003/chasseriaupeyon/article.htm

21. C. Gérardot, loc. cit., p. 76.

22. S. Landrin, « Lyon redonne vie à sa presqu'île entre Saône et Rhône ", Le Monde, $1^{\text {er }}$ avril 2005.

23. De 1960 à 2002, alors que l'agglomération passait de 360000 à 555000 habitants, la surface urbanisée a été plus que triplée. Le rapport du nombre d'habitants par surface est ainsi passé de 72 hab./ha en 1960 à 51 hab./ha en 1975 et 35 hab./ha en 2002.

24. A. Masboungi, Nantes - La Loire dessine le projet, Projet urbain, Éditions La Villette, DGUHC, 2003.

25. A. Chemetoff, in « Nantes demain », 2005, p. 35.

26. A. Masboungi, op. cit., p. 131.

27. C. Gérardot, loc. cit., p. 77.

28. Ibid., p. 78.

29. D. Chiappero, « Patrimoine ligérien et développement urbain : définition et nouvelles pratiques d'aménagement », thèse de géographie, Université de Tours, 1996.

\section{RÉSUMÉS}

Fleuve et ville forment un couple selon un genre qui n'est pas nouveau, même s'il a été ravivé ces dernières décennies, à la fois par des discours et par des projets : la première idée pour expliquer ce revival est la montée des préoccupations pour la préservation de la nature et du paysage, voire, plus extrémiste encore, la montée d'un désir de voir s'améliorer les conditions écologiques, esthétiques et sociales de ces milieux très abîmés. Cet article examine donc dans quelle mesure l'écologie est invoquée dans les opérations de réappropriation des fleuves par les villes. Comment le fleuve est-il travaillé dans ces projets de « reconquête »? Est-ce une valorisation esthétique, symbolique ou écologique? ou l'émergence de nouvelles pratiques sociales en milieu urbain? Nous nous appuyons sur de nombreux exemples européens, mais une partie se penche particulièrement sur la place du fleuve dans deux projets urbains, les îles du Rhône en aval de Lyon ; et l'île de Nantes, sur la Loire. Cela nous conduit notamment à infirmer l'hypothèse d'un lien entre cette dynamique et une montée des préoccupations pour les hydroécosystèmes comme milieu de vie (êtres humains inclus), en ville. La valorisation du fleuve en ville prend place effectivement au titre d'un visuel, et d'une symbolique vectrice d'identité. Mais le chemin qui conduirait à un fonctionnement écologique de la ville au bord de son fleuve, à une harmonie entre images et usages du fleuve, s'il existe, est encore long.

Sophie Bonin, Rivers and the city : ecological issues and urban projects

River and city form a couple of a kind which is not new, even if it has been revived in past decades, at the same time in discourses and projects: the first idea to explain this revival is the growing concern for nature and landscape protection and even more, the rise of a desire to improve the ecological, aesthetic and social conditions of these ecosystems. This article thus examines the extent to which ecology is called upon in the operations of reconquest of the rivers by the cities. How is the river dealt with in these projects of "reconquest"? Does this correspond to an aesthetic, symbolic, or ecological valorisation, or to the emergence of new social practices 
in urban environment? The analysis is based on several european examples, focusing particularly on the place of the river in two urban projects, the islands of the Rhone downstream from Lyon; and the île de Nantes, on the Loire. These latter examples lead us to refute the assumption of a relationship between this dynamics and a growing interest in hydroecosystems as a life environment (including for human beings). The value given to the downtown river is indeed associated with a visual and symbolic system operating as a vector of identity. But it would take a long way to achieve the goals of and ecological functioning of the city at the edge of the river, reconciling, if possible, between images and uses of the river.

INDEX

Mots-clés : Paysage, paysage urbain, fleuve, écologie urbaine, milieu de vie, projets urbains Keywords : landscape, urban landscape, river, urban ecology, medium of life, urban projects

\section{AUTEUR}

\section{SOPHIE BONIN}

Maître de conférence en géographie, IGA-UMR PACTE-Territoires : 14 bis, avenue Marie-Reynoard 38100 Grenoble, sofie.bonin@free.fr 